\title{
Number of Lymph Nodes with Macrometastases
}

National Cancer Institute

\section{Source}

National Cancer Institute. Number of Lymph Nodes with Macrometastases. NCI

Thesaurus. Code C147904.

The number of examined lymph nodes that were positive for macrometastases. 